\title{
Impact of propofol sedation versus opioid/benzodiazepine sedation on colonoscopy outcomes: a systematic review with meta-analysis
}

\section{(잉 $\odot$}

\section{Authors}

Muhammad Aziz¹, Simcha Weissman², Rawish Fatima1, Zubair Khan ${ }^{3}$, Babu P. Mohan4, Tej I. Mehta ${ }^{5}$, Wade Lee-Smith ${ }^{6}$, Ammar Hassan? $^{7}$, Michael Sciarra7 ${ }^{7}$, Ali Nawras ${ }^{8}$, Douglas G. Adler ${ }^{9}$

Institutions

1 Department of Internal Medicine, University of Toledo Medical Center, Toledo, Ohio, United States

2 Department of Medicine, Hackensack University Palisades Medical Center, North Bergen, New Jersey, United States

3 Department of Gastroenterology, McGovern Medical School, University of Texas Health Science Center at Houston, Houston, Texas, United States

4 Department of Internal Medicine, Banner University Medical Center Tucson, Arizona, United States

5 Department of Medicine, University of South Dakota Sanford School of Medicine, Sioux Falls, South Dakota, United States

6 University Libraries, University of Toledo, Ohio, United States

7 Division of Gastroenterology, Hackensack University Palisades Medical Center, North Bergen, New Jersey, United States

8 Department of Gastroenterology, University of Toledo, Toledo, Ohio, United States

9 Department of Gastroenterology, University of Utah, Salt Lake City, Utah, United States

submitted 29.10.2019

accepted after revision $\quad 2.1 .2020$

Bibliography

DOI https://doi.org/10.1055/a-1135-8681 |

Endoscopy International Open 2020; 08: E701-E707

(c) Georg Thieme Verlag KG Stuttgart · New York

eISSN 2196-9736

\& Supplementary material

Online content viewable at:

https://doi.org/10.1055/a-1135-8681
Corresponding author

Douglas G. Adler, MD, University of Utah, Salt Lake City, Utah

Fax: +1-801-581-8007

Douglas.Adler@hsc.utah.edu

\section{ABSTRACT}

Background and study aims Choice of sedation (propofol vs opioid/benzodiazepine) has been studied in the literature and has shown variable outcomes. The majority of recent studies have evaluated propofol sedation (PS) versus opioids, benzodiazepines, or a combination of both. We performed a systematic review and meta-analysis of studies comparing PS to other sedation methods to assess the impact on colonoscopy outcomes.

Methods Multiple databases were searched and studies of interest were extracted. Primary outcome of the study was adenoma detection rate (ADR) and secondary outcomes included polyp detection rate (PDR), advanced adenoma detection rate (AADR), and cecal intubation rate (CIR).

Results A total of 11 studies met the inclusion criteria with a total of 177,016 patients $(148,753$ and 28,263 in the opioids/benzodiazepine group and PS group, respectively). Overall, ADR (RR: 1.07, 95\% Cl 0.99-1.15), PDR (RR: 1.01, $95 \% \mathrm{Cl} 0.93-1.10$ ), and AADR (RR: $1.17,95 \% \mathrm{Cl} 0.92-$ 1.48 ) did not improve with the use of PS. The CIR was slightly higher for propofol sedation group (RR 1.02, $95 \% \mathrm{Cl}$ 1.00-1.03).

Conclusion Based on our analysis, PS and opioid/benzodiazepine sedation seem to have comparable ADR. Our results do not favor use of a particular sedation method and the choice of sedation should be individualized based on patient preference, risk factors and resource availability. 


\section{Introduction}

Colonoscopy remains a widely performed diagnostic modality of screening for colorectal cancer. Detection and subsequent removal of colonic polyps (most notably adenomatous polyps) has been shown to decrease incidence of colorectal cancer [1]. Adenomatous polyps can be missed in up to a quarter of colonoscopies and hence multiple gastrointestinal societies have proposed quality indicators or metrics to uphold competency in colonoscopy [2-4]. These include adenoma detection rate $(A D R)$, polyp detection rate (PDR), advanced adenoma detection rate (AADR) and cecal intubation rate (CIR).

Currently, two methods of sedation are most commonly used to achieve patient relaxation and thus compliance during the colonoscopy exam: opioid/benzodiazepine sedation (OBS) i. e. conscious sedation, as well as propofol-based sedation (PS) [5]. Use of OBS generally entails gastroenterologist-monitored use of an opioid and a benzodiazepine or a combination of both agents while PS, which can be administered via an anesthesiologist, a nurse anesthetist, or a nurse working closely with the endoscopists, has been increasingly utilized recently to achieve a deeper level of sedation to further increase patient satisfaction. $[1,5]$

A recent meta-analysis that compared PS with OBS did not reveal any statistically significant increase in patient satisfaction $(R R=0.94,95 \% C l 0.86-1.04)$, physician satisfaction $(R R=$ $0.35,95 \% \mathrm{Cl} 0.02-6.95$ ), and recovery time (mean difference $=-6.77,95 \% \mathrm{Cl}-16.21-2.67)$ [5]. Randomized controlled trials (RCTs) and cohort studies comparing use of OBS vs PS for colonoscopy have shown varying results in terms of differences in ADR, PDR, AADR, and CIR [6-15]. To further clarify these outcomes, we conducted a systematic review and meta-analysis of these studies to determine whether PS in comparison to OBS improves the aforementioned quality metrics.

\section{Methods}

\section{Study definitions}

ADR was defined as patients with one or more adenomas detected on colonoscopy of all the patients undergoing colonoscopy. Advanced adenomas (AA) are defined as adenomas $\geq 10$ $\mathrm{mm}$ or those with high-grade dysplasia and/or villous/adenocarcinoma component on histology. AADR was defined as patients with one or more advanced adenomas detected on colonoscopy.

\section{Search strategy}

A comprehensive literature search was performed using the following databases: PubMed/Medline, Embase, Cochrane Register of Controlled Trials, Web of Science Core Collection, and CINAHL from inception through July 30, 2019 to identify all relevant articles. Controlled subject terms and keyword synonyms for the concepts of colonoscopy, adenoma/polyp detection rate and propofol sedation were developed for PubMed and translated to the vocabularies and syntax of the other databases. The search strategy was created and performed by a librarian (W.L.S.) and cross checked by another reviewer (M.A.). Bib- liographic references for included articles were also screened. Relevant articles for final data extraction were shortlisted by two reviewers (M.A. and S.W.). PRISMA and MOOSE guidelines were used to select the final articles. The detailed search strategy for PubMed is highlighted in Supplementary Table 1.

\section{Inclusion and exclusion criteria}

Our search strategy was limited to RCTs and cohort studies only. We excluded all other studies including editorials, case reports, case series, and single arm studies. The search strategy was not restricted to language or dates. We included all relevant abstracts as well.

\section{Data collection}

Baseline demographic data (age, sex, ethnicity), colonoscopy indication (screening/surveillance vs diagnostic), and outcomes (ADR, PDR, AADR, and CIR) were extracted where applicable.

\section{Primary and secondary outcomes}

The primary outcome of our analysis was ADR and secondary outcomes included PDR, AADR and CIR for PS and OBS group. Subgroup analysis was performed if there was at least one study available for a particular specific sedation drug or combination. Further, subgroup analysis was performed for full-length articles only.

We generated the outcomes on the basis of intention-totreat (ITT) analysis for RCTs. This means that outcomes were generated for all patients on the basis of randomization even if they did not complete the study for any reason.

\section{Data synthesis and statistical analysis}

Data were extracted using Microsoft Excel (Microsoft, Redmond, Washington, United States). Pooled proportion rates for all outcomes were compared using risk ratio (RR) with $95 \%$ confidence interval $(\mathrm{Cl}) .95 \%$ prediction intervals (PIs) are also reported. The outcomes were calculated using the DerSimonian-Laird method and random effects model. The 12 statistic was used to evaluate heterogeneity between the studies as defined by Cochrane handbook for systematic reviews $[16,17]$. Open Meta Analyst (CEBM, University of Oxford, Oxford, United Kingdom) was used as the computing software for generating all outcomes. $P<0.05$ was considered statistically significant when comparing proportions between the two groups. Subgroup analysis based on specific sedation type and meta-regression controlling factors such as age, gender, and study type was applied to see if any statistical difference existed.

\section{Bias assessment}

Study quality was assessed using Cochran Risk of bias tools for RCTs and Newcastle Ottawa score for cohort studies [18, 19]. Publication bias is displayed using funnel plots that were generated using Review Manager V5.3 (The Cochrane Collaboration, Oxford, United Kingdom). 


\section{Results}

A total of 123 studies were retrieved after eliminating duplicates based on our search strategy ( $\triangleright$ Fig. 1 ). A total of 11 studies ( 8 full-length articles and 3 abstracts) met inclusion criteria.

Of the finalized studies, one was a RCT [8] and 10 were cohort studies [1, 6, 7, 9-15]. Risk of bias assessment is shown in Supplementary Table 2 and Supplementary Table 3 for RCT and cohort studies, respectively. The RCT had a high risk of bias due to the impractical nature of blinding of the endoscopist during the procedure. The cohort studies had a score of $\geq 4$ (maximum 6) on Newcastle-Ottawa scale. No visible asymmetrical distribution of studies were noted on funnel plot for publication bias based on adenoma detection rate. (Supplementary Fig. 1).

Study details and demographics are summarized in Supplementary Table 4. Eight studies used midazolam and fentanyl specifically for OBS.[1, 6, 9-13, 15] The total number of patients in both groups was 177,016 (148753 and 28263 in OBS and PS groups, respectively). Both groups (OBS vs PS) were similar in terms of age (range 55.4-64 and 56.7-67) and male gender (53.2\% vs 50.9\%).

Primary and secondary outcomes are shown in Supplementary Table 5.

\section{Adenoma detection rate}

Overall seven studies reported ADR $[1,8,10-12,14,15]$. ADR was not significantly higher for the PS group compared to the OBS group (RR: 1.07, $95 \% \mathrm{Cl} 0.99-1.15, P=0.09, \mathrm{I} 2=27.3 \%, \mathrm{PI}$ 0.82-1.24) ( Fig. 2a). Neither subgroup analysis by study type nor meta-regression by median age or gender distribution significantly affected these results. A further subgroup analysis of six studies that used a combination of fentanyl and midazolam for OBS demonstrated similar results (RR: 1.07 , $95 \% \mathrm{Cl} 0.98-$ 1.16, $P=0.11, I 2=37.3 \%$, PI 0.93-1.30)( Fig. 2b).

\section{Polyp detection rate}

Six studies assessed PDR and no statistically significant difference was found between the two groups (RR: $1.01,95 \% \mathrm{Cl}$ $0.93-1.10, P=0.83, I^{2}=91.2 \%, P I 0.82-1.24$ ) ( Fig. 3a) $[6,7$, $9,12-13]$. Four studies used the fenanyl/midazolam combination for OBS and demonstrated no statistical difference as well (RR: $1.00,95 \% \mathrm{Cl} 0.90-1.11, P=0.99, \mathrm{I}^{2}=93.1 \%$, PI 0.81-1.24) ( $\triangleright$ Fig.3b). Subgroup analysis by study type did not significantly affect these results.

\section{Advanced ADR}

Three studies assessed AADR and no statistically significant difference was noted (RR: 1.17, $95 \% \mathrm{Cl} 0.92-1.48, \mathrm{p}=0.21, \mathrm{I}^{2}=$ $61.6 \%$, PI 0.36-3.03) ( Fig. 4) $[11,13,14]$.

\section{CIR}

CIR was assessed in five studies and significantly increased intubation rates were found in PS group compared to OBS group (RR: 1.02 , 95\% Cl $1.00-1.03, P=0.047, I^{2}=92.9 \%$, PI $0.98-$ 1.05) ( Fig. 5a) $[6,7,11,13,15]$. Four studies used fentanyl/ midazolam combination for OBS, and increased CIR was found
195 records identified through database searching

28 in Pubmed

33 in Cochrane Library

102 in Embase

27 in Web of Science

5 in CINAHL

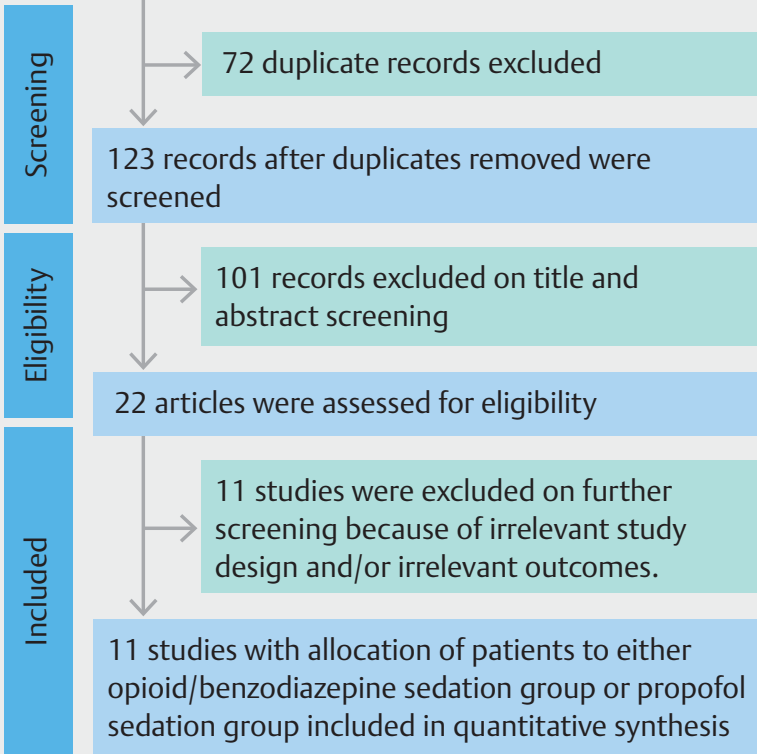

- Fig. 1 Flow diagram representing the selection of studies.

in PS group (RR: $1.02,95 \% \mathrm{Cl} 1.00-1.04, P=0.02, \mathrm{I}^{2}=93.8 \%, \mathrm{PI}$ 0.99-1.05) (- Fig. 5c).

\section{Subgroup analysis}

A subgroup analysis of RCT versus non-RCT studies and abstracts versus full studies are summarized in $>$ Table 1 . Only CIR showed significant results when analysis was restricted to non-RCTs and manuscripts (RR: $1.02,95 \% \mathrm{Cl} 1.00-1.03, P=$ 0.047 and RR: $1.02,95 \% \mathrm{Cl} 1.00-1.04, P=0.048)$. Despite the statistical significance, the actual difference in CIR was minimal and clinically not relevant. Interestingly AADR was higher when analysis was restricted to full manuscripts only (RR: 1.24, $95 \%$ $\mathrm{Cl} 1.08-1.48, P=0.003)$. No significant difference was observed when studies were collectively analyzed to assess PDR and ADR.

\section{Discussion}

Our systematic review and meta-analysis demonstrates that colonoscopy performed under PS did not improve ADR, PDR, or AADR compared to OBS group. We did find slightly higher CIR with PS method. The results were consistent when subgroup analysis was performed for studies that strictly used the combination of fentanyl and midazolam for OBS.

High-quality colonoscopy requires an adequate level of patient sedation and subsequent relaxation, which are both pertinent for optimal mucosal evaluation and key clinical outcomes [6]. Historically, sedation was achieved by administration of a 


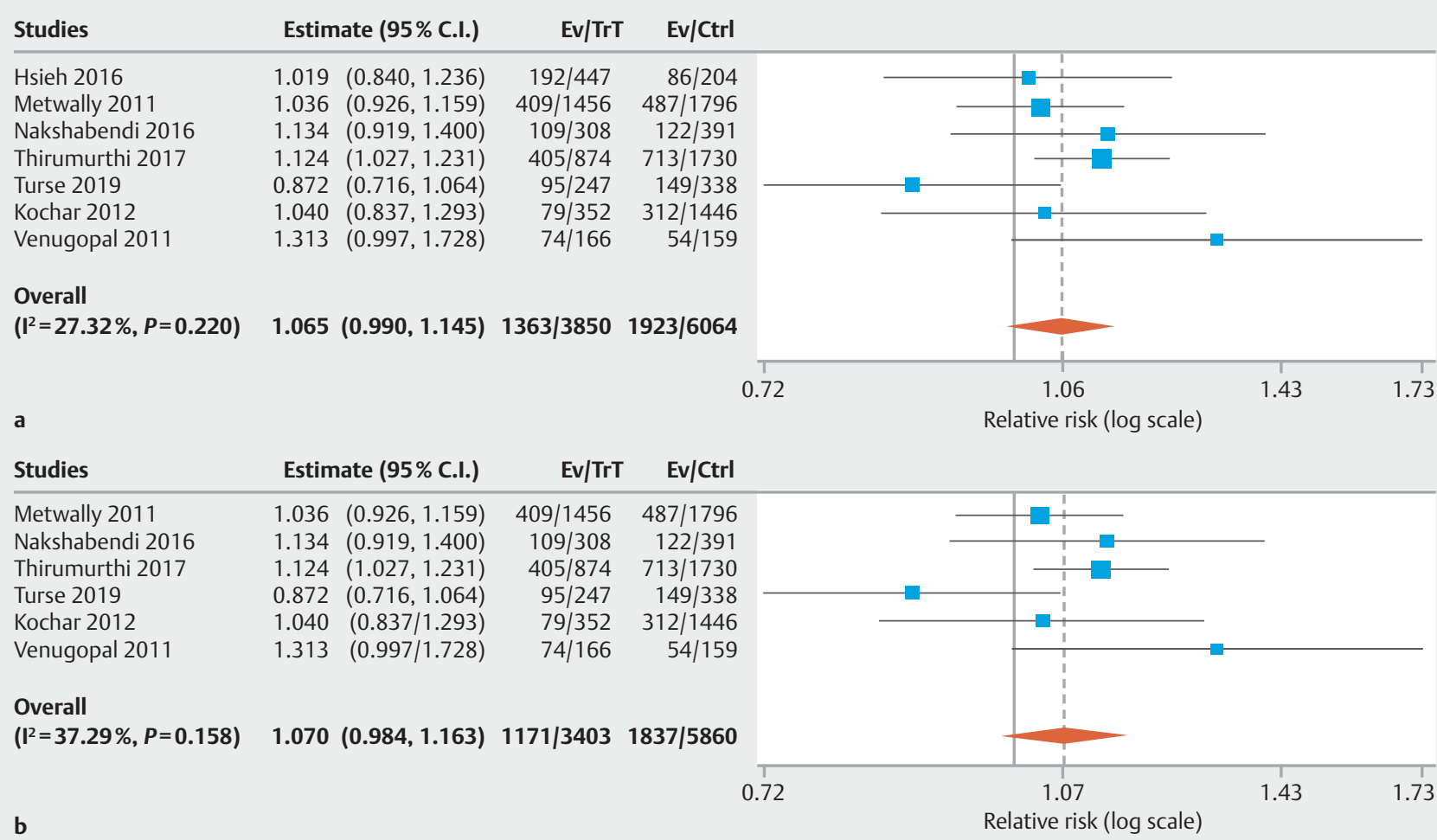

- Fig. 2 Forest plot comparing adenoma detection rate in PS versus OBS group. a Overall. b Fentanyl/midazolam combination. (C.I, confidence interval; PS, propofol sedation; OBS, opioid/benzodiazepine sedation; Trt, PS group; Ctrl, OBS group).

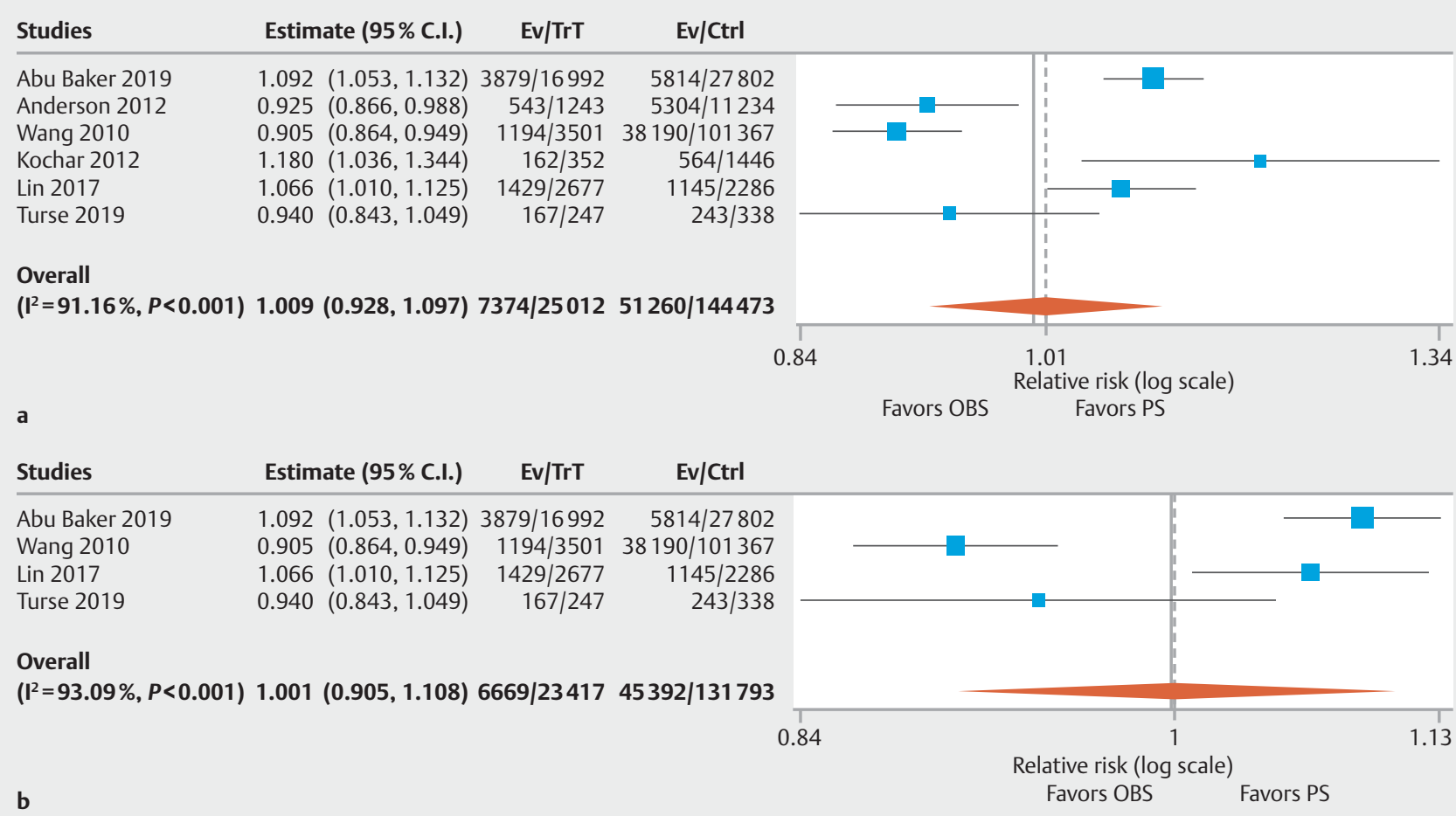

- Fig. 3 Forest plot comparing polyp detection rate in PS versus OBS group. a Overall. b Fentanyl/midazolam combination. C.I, confidence interval; PS, propofol sedation; OBS, opioid/benzodiazepine sedation; Trt, PS group; Ctrl, OBS group). 


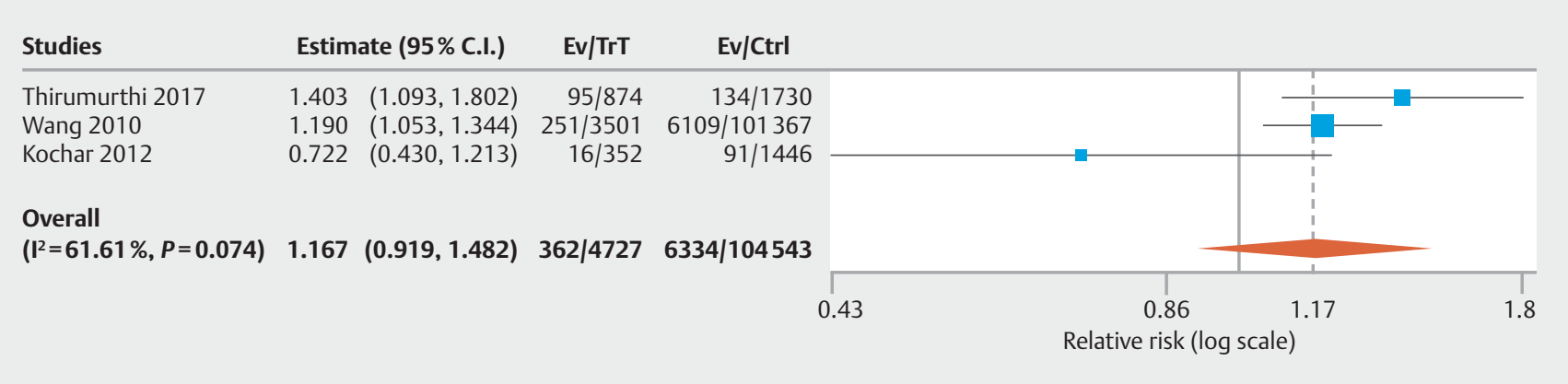

- Fig. 4 Forest plot comparing overall advanced adenoma detection rate in PS versus OBS group. (C.I, confidence interval; PS, propofol sedation; OBS, opioid/benzodiazepine sedation; Trt, PS group; Ctrl, OBS group).

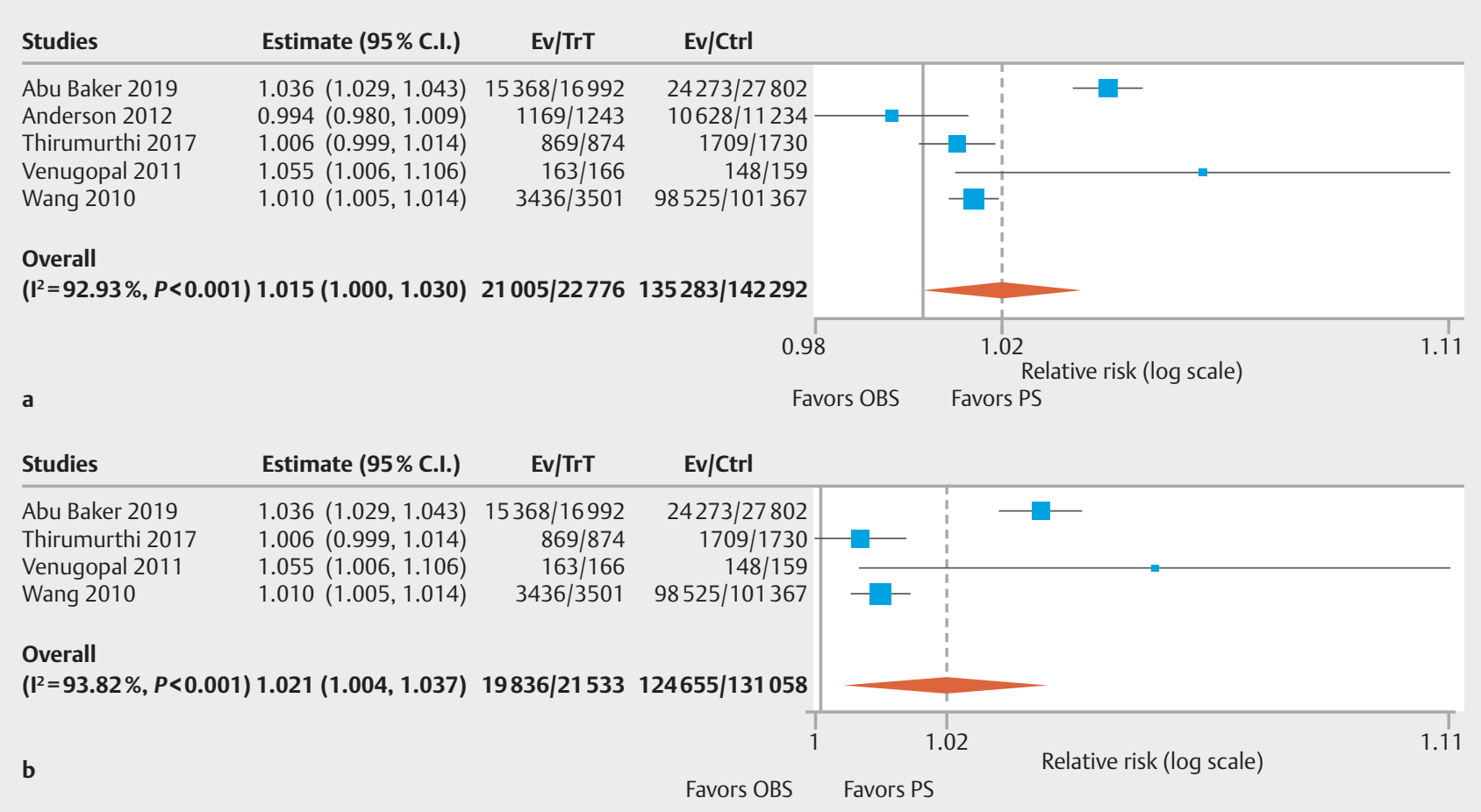

- Fig. 5 Forest plot comparing Cecal Intubation rate in PS versus OBS group. a Overall. b Fentanyl/midazolam combination. (C.I, confidence interval; PS, propofol sedation; OBS, opioid/benzodiazepine sedation; Trt, PS group; Ctrl, OBS group).

combination of benzodiazepines and opioids, which allowed the patient to obtain a moderate level of sedation [6]. However, more recently, owning to its rapid onset of action and its favorable safety profile, propofol-based sedation, or PS, has been introduced as an alternative $[6,20]$.

There are a number of benefits of utilizing propofol for sedation during colonoscopy. Firstly, PS appears to result in greater patient satisfaction [20]. Next, in light of increased patient comfort, improved patient compliance and adherence to national colorectal cancer screening guidelines should, in theory, follow. Finally, based on our results as well, there is a higher likelihood that cecal or terminal ileum intubations can be performed and that the examination can be completed [21]. Still, there are no clearly established effects as to the efficiency of PS which can justify its widespread use. Its increased cost and thus higher economic healthcare burden is a reasonable concern [6, 22]. In addition, the setting and personnel required to provide PS, can limit its availability in certain endoscopic centers [5, 6]. Potential utilization of nurse-administered propofol sedation (NAPS) method as pointed out by Rex et al. can address both major concerns i.e. high cost and safe administration of the drug $[23,24]$. Another concern with the widespread use of PS is increased risk of aspiration pneumonia as highlighted by Bielawska et al in a population-based study [25].

Our study included one RCT by Hsieh et al. which demonstrated slightly higher ADR for the PS compared to meperidine group (OBS) (43.0\% vs $42.2 \%$ respectively), however their comparison did not achieve statistical significance $(P=0.85)$ [8]. The authors had a different objective i. e. comparison of ADR in patients undergoing air insufflation, water immersion, and water 
- Table 1 Sensitivity analysis on the basis of study design (RCT vs non-RCT and abstracts vs full manuscripts).

\begin{tabular}{|c|c|c|c|c|c|c|c|}
\hline Outcome & Subgroup & $\begin{array}{l}\text { No. of studies } \\
\text { Based on } \\
\text { study design }\end{array}$ & $I^{2}$ & $P$ value & RR $(95 \% \mathrm{Cl})$ & $P$ value & Overall $P$ value \\
\hline \multirow[t]{4}{*}{ ADR } & $\mathrm{RCT}$ & 1 & NA & NA & NA & NA & \multirow[t]{4}{*}{0.09} \\
\hline & Non-RCT & 6 & $37.3 \%$ & 0.11 & $1.07(0.98-1.16)$ & 0.11 & \\
\hline & Abstracts & 2 & $40.8 \%$ & 0.19 & $1.15(0.92-1.44)$ & 0.23 & \\
\hline & Manuscripts & 5 & $33.7 \%$ & 0.20 & $1.05(0.97-1.14)$ & 0.23 & \\
\hline \multirow[t]{4}{*}{ PDR } & $\mathrm{RCT}$ & 0 & NA & NA & NA & NA & \multirow[t]{4}{*}{0.83} \\
\hline & Non-RCT & 6 & $91.2 \%$ & $<0.01$ & $1.01(0.93-1.10)$ & 0.83 & \\
\hline & Abstracts & 2 & $90.6 \%$ & $<0.01$ & $1.04(0.82-1.32)$ & 0.76 & \\
\hline & Manuscripts & 4 & $93.1 \%$ & $<0.01$ & $1.00(0.91-1.11)$ & 0.99 & \\
\hline \multirow[t]{4}{*}{ AADR } & $\mathrm{RCT}$ & 0 & NA & NA & NA & NA & \multirow[t]{4}{*}{0.21} \\
\hline & Non-RCT & 3 & $61.6 \%$ & 0.07 & $1.17(0.92-1.48)$ & 0.21 & \\
\hline & Abstracts & 1 & NA & NA & NA & NA & \\
\hline & Manuscripts & 2 & $26.3 \%$ & 0.24 & $1.24(1.08-1.48)$ & 0.003 & \\
\hline \multirow[t]{4}{*}{ CIR } & $\mathrm{RCT}$ & 0 & NA & NA & NA & NA & \multirow[t]{4}{*}{0.047} \\
\hline & Non-RCT & 5 & $92.9 \%$ & $<0.01$ & $1.02(1.00-1.03)$ & 0.047 & \\
\hline & Abstracts & 2 & $82.0 \%$ & 0.02 & $1.02(0.96-1.08)$ & 0.51 & \\
\hline & Manuscripts & 3 & $95.7 \%$ & $<0.01$ & $1.02(1.00-1.04)$ & 0.048 & \\
\hline
\end{tabular}

exchange colonoscopy and the method of sedation utilized was evaluated as a subgroup analysis. Our meta-analysis did not show improved ADR for either sedation groups which was consistent with the study by Hsieh et al.

Although colonoscopy is the gold standard for colorectal cancer screening, its miss rate for cancer detection, albeit low, remains a genuine concern [3]. Efforts are directed to improve the ADR which is the most important independent predictor of risk of interval colorectal cancer after screening colonoscopy $[2,6,26]$. In the current study, given that patients undergoing colonoscopy with PS did not show a statistically significantly higher ADR and PDR than patients undergoing OBS, and the added risk of aspiration pneumonia, use of PS versus OBS should be individualized based on patient preference, risk factors, and resource availability.

The main limitation of this meta-analysis is that only one RCT was available. In an attempt to circumvent this limitation, we included non-randomized trials. However, the inclusion of studies with non-randomized design introduces possible significant selection bias and heterogeneity. Second, no standardized dosing for sedation medications was observed and the level of sedation achieved was based on a subjective judgement by the caregivers at the time of the exam. Another limitation was inconsistent use of colonoscopy equipment as certain endoscopy centers had high-definition colonoscopy with distal attachments and/or electronic chromoendoscopy. Inclusion of pa- tients other than for a screening/surveillance indication as well as presence of more white patients in the study can alter the overall outcome metrics such as PDR and ADR, thus the results should be carefully interpreted based on this. Further, none of the studies reported data on serrated adenoma detection, which is another important colonoscopy outcome metric. Lastly, endoscopist expertise and self-reporting data are subjective and the possibility of non-random user-error remains. These limitations are, at least in part, attenuated by the combined statistical power of a large number of studies with a vast and diverse study population included in the proportional meta-analysis. In addition, we set forth strict inclusion and exclusion criteria in an effort to limit bias.

\section{Conclusion}

In conclusion, use of PS and/or OBS during colonoscopy was associated with comparable ADR, PDR and AADR. Although, PS did result in higher CIR, this association was weak. Future research, specifically RCTs, are needed to comment on the statistical significance of these quality metrics during PS. There is also a need to assess serrated adenoma detection rate with the use of PS compared to OBS. These data will help further guide appropriate recommendations for use of PS during colonoscopy. 


\section{Competing interests}

The authors declare that they have no conflict of interest.

References

[1] Metwally M, Agresti N, Hale WB et al. Conscious or unconscious: The impact of sedation choice on colon adenoma detection. World J Gastroenterol 2011; 17: 3912-3915

[2] Borg BB, Gupta NK, Zuckerman GR et al. Impact of obesity on bowel preparation for colonoscopy. Clin Gastroenterol Hepatol 2009; 7: 670-675

[3] Rex DK, Cutler CS, Lemmel GT et al. Colonoscopic miss rates of adenomas determined by back-to-back colonoscopies. Gastroenterology 1997; 112: 24-28

[4] Rex DK, Petrini JL, Baron TH et al. Quality indicators for colonoscopy. Gastrointest Endoscopy 2006; 63: S16-S28

[5] Lim S, Lee OH, Yoon IJ et al. Moderate versus deep sedation in adults undergoing colonoscopy: systematic review and meta-analysis. Curr Med Res Opin 2019; 35: 879-885

[6] Baker FA, Mari A, Amarney K et al. Propofol sedation in colonoscopy: from satisfied patients to improved quality indicators. Clin Exp Gastroenterol 2019; 12: 105-110

[7] Anderson JC, Weiss JE, Goodrich M et al. Tu1719 does propofol use affect colonoscopy outcomes? Data from New Hampshire Colonoscopy Registry Gastrointest Endosc 2012: 75

[8] Hsieh Y-H, Tseng C-W, Hu C-T et al. Prospective multicenter randomized controlled trial comparing adenoma detection rate in colonoscopy using water exchange, water immersion, and air insufflation. Gastrointest Endosc 2017; 86: 192-201

[9] Lin OS, Selva DL, Kozarek RA et al. One year experience with computer-assisted propofol sedation for colonoscopy. World J Gastroenterol 2017; 23: 2964-2971

[10] Nakshabendi R, Berry AC, Munoz JC et al. Choice of sedation and its impact on adenoma detection rate in screening colonoscopies. Ann Gastroenterol 2016; 29: 50-55

[11] Thirumurthi S, Raju GS, Pande M et al. Does deep sedation with propofol affect adenoma detection rates in average risk screening colonoscopy exams? World J Gastrointest Endosc 2017; 9: 177-182

[12] Turse EP, Dailey FE, Bechtold ML. Impact of moderate versus deep sedation on adenoma detection rate in index average-risk screening colonoscopies. Gastrointest Endosc 2019; 90: 502-505
[13] Wang A, Hoda KM, Holub JL et al. Does Level of Sedation Impact Detection of Advanced Neoplasia? Dig Dis Sci 2010; 55: 2337-2343

[14] Kochar R, Guturu P, Soloway RD et al. Su1494 Moderate sedation compared with anesthesiology administered propofol sedation in polyp and adenoma detection during screening colonoscopy. Gastrointest Endosc 2012: 75

[15] Venugopal K, Pearce CB. Colonoscopy with propofol sedation: better for the patient and for the endoscopist. J Gastroenterol Hepatol 2011; 26: (Suppl. 04): 22-33

[16] Higgins ], Thompson SG, Deeks J] et al. Measuring inconsistency in meta-analyses. BMJ 2003; 327: 557-560

[17] Shuster J. Review: Cochrane handbook for systematic reviews for interventions, Version 5.1.0, published 3/2011. Julian P.T. Higgins and Sally Green, Editors. Res Synth Methods 2011; 2: 126-130

[18] Higgins J, Altman D, Gotzsche P et al. The Cochrane Collaboration's tool for assessing risk of bias in randomised trials. BMJ 2011; 343: d5928-d5928

[19] Deeks J, Dinnes J, D'Amico R et al. Evaluating non-randomised intervention studies. Health Technol Assess 2003; 7: iii-x, 1-173

[20] Padmanabhan A, Frangopoulos C, Shaffer LET. Patient satisfaction with propofol for outpatient colonoscopy: a prospective, randomized, double-blind study. Dis Colon Rectum 2017; 60: 1102-1108

[21] Radaelli F, Meucci G, Sgroi G et al. Italian Association of Hospital Gastroenterologists (AIGO). Technical performance of colonoscopy: the key role of sedation/analgesia and other quality indicators. Am J Gastroenterol 2008; 103: 1122-1130

[22] Pace D, Borgaonkar M. Deep sedation for colonoscopy is unnecessary and wasteful. CMAJ 2018; 190: E153-E154

[23] Rex DK, Overley C, Kinser K et al. Safety of propofol administered by registered nurses with gastroenterologist supervision in 2000 endoscopic cases. Am J Gastroenterol 2002; 97: 1159-1163

[24] Rex DK, Heuss LT, Walker JA et al. Trained registered nurses/endoscopy teams can administer propofol safely for endoscopy. Gastroenterology 2005; 129: 1384-1391

[25] Bielawska B, Hookey LC, Sutradhar R et al. Anesthesia assistance in outpatient colonoscopy and risk of aspiration pneumonia, bowel perforation, and splenic injury. Gastroenterology 2018; 154: 77-85

[26] Kaminski MF, Regula J, Kraszewska E et al. Quality indicators for colonoscopy and the risk of interval cancer. N Engl ] Med 2010; 362: 1795-1803 